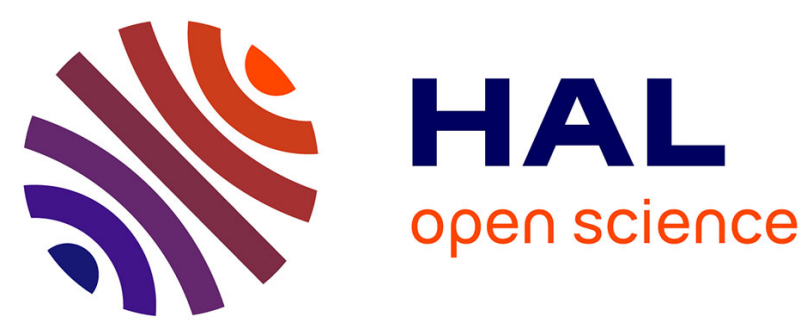

\title{
Evidence of a C60 / Co interface reconstruction and its influence on magnetic properties
}

\author{
Cynthia Fourmental, Ludovic Le Laurent, Vincent Repain, Cyril Chacon, \\ Yann Girard, Jérôme Lagoute, Sylvie Rousset, Alessandro Coati, Yves \\ Garreau, Andrea Resta, et al.
}

\section{To cite this version:}

Cynthia Fourmental, Ludovic Le Laurent, Vincent Repain, Cyril Chacon, Yann Girard, et al.. Evidence of a C60 / Co interface reconstruction and its influence on magnetic properties. Physical Review B, 2021, 104 (23), pp.235413. 10.1103/PhysRevB.104.235413 . hal-03540843

\section{HAL Id: hal-03540843 https://hal.science/hal-03540843}

Submitted on 24 Jan 2022

HAL is a multi-disciplinary open access archive for the deposit and dissemination of scientific research documents, whether they are published or not. The documents may come from teaching and research institutions in France or abroad, or from public or private research centers.
L'archive ouverte pluridisciplinaire HAL, est destinée au dépôt et à la diffusion de documents scientifiques de niveau recherche, publiés ou non, émanant des établissements d'enseignement et de recherche français ou étrangers, des laboratoires publics ou privés. 


\title{
Evidence of $\mathrm{a}_{60} / \mathrm{Co}$ interface reconstruction and its influence on magnetic properties
}

\author{
Cynthia Fourmental, ${ }^{1}$ Ludovic Le Laurent, ${ }^{2}$ Vincent Repain,,${ }^{1, *}$ Cyril Chacon, ${ }^{1}$ Yann Girard $\odot,{ }^{1}$ Jérôme Lagoute, ${ }^{1}$ \\ Sylvie Rousset, ${ }^{1}$ Alessandro Coati, ${ }^{3}$ Yves Garreau $\odot,{ }^{3,1}$ Andrea Resta $\odot,{ }^{3}$ Alina Vlad, ${ }^{3}$ Cyrille Barreteau, ${ }^{2}$ \\ Alexander Smogunov, ${ }^{2}$ Dongzhe Li $\odot,{ }^{4}$ and Amandine Bellec ${ }^{1}{ }^{1}$ \\ ${ }^{1}$ Université de Paris, CNRS, Laboratoire Matériaux et Phénomènes Quantiques, 10, rue A. Domon et L. Duquet, 75013 Paris, France \\ ${ }^{2}$ SPEC, CEA, CNRS, Université Paris-Saclay, CEA Saclay, 91191 Cedex Gif-sur-Yvette, France \\ ${ }^{3}$ Synchrotron SOLEIL, L'Orme des Merisiers, Saint-Aubin, 91192 Gif sur Yvette, France \\ ${ }^{4}$ CEMES, CNRS, Université de Toulouse, 29 rue Jeanne Marvig, F-31055 Toulouse, France
}

(Received 10 July 2021; revised 19 October 2021; accepted 29 November 2021; published 10 December 2021)

\begin{abstract}
By using grazing incidence $\mathrm{x}$-ray diffraction and scanning tunneling microscopy experiments, we determine the interface structure of a $\mathrm{C}_{60}$ layer deposited on a $\mathrm{Co}(0001)$ surface. The room temperature deposition gives rise to a $(4 \times 4)$ molecular structure with a $10 \%$ vertical relaxation of the underneath Co atoms. When annealed around $550 \mathrm{~K}$, the interface undergoes a structural change with most of the buckyballs sitting in a seven Co vacancies nanohole. Ab initio calculations show that this interface reconstruction is stabilized by a strong C-Co hybridization and has a significant impact both on the interfacial magnetic anisotropy that is decreased and on the molecular spin polarization that it increased.
\end{abstract}

DOI: 10.1103/PhysRevB.104.235413

\section{INTRODUCTION}

The use of molecular materials in spintronic devices has been growing over the past decade, leading to the observation of unexpected properties like giant spin polarization [1] or interface magnetoresistance [2]. Among other molecules, $\mathrm{C}_{60}$ has been one of the most studied with the achievement of significant room temperature magnetoresistance, in combination with metallic ferromagnetic electrodes like Co [3]. The weak spin-orbit coupling and hyperfine interaction of this molecule are particularly interesting to obtain a long spindiffusion length of typically larger than $100 \mathrm{~nm}$ [4]. However, magnetotransport results with these molecular materials generally lack reproducibility and smaller magnetoresistance values have also been reported with similar samples, possibly due to a less efficient spin injection [5]. It seems that the ferromagnet/molecule interface plays a key role in the transport properties [1] and tuning the so-called spinterface has become an important challenge in this field [6].

Surprisingly, not only the spin polarization but also the magnetic anisotropy has been found deeply modified at the molecule/ferromagnet interface. For example, the $\mathrm{C} p_{z}-\mathrm{Co}$ $d_{z^{2}}$ hybridization has been demonstrated to be at the origin of an enhanced perpendicular anisotropy at the $\mathrm{C}_{60} / \mathrm{Co}(0001)$ interface $[7,8]$, which induces a magnetic hardening in either out-of-plane [9] or in-plane magnetized samples [10]. The link between magnetic properties and the fine details of the interface structure has not been studied extensively up to now for such samples even if few experimental evidences have been reported, either on the magnetic [11] or the transport properties [12].

\footnotetext{
*vincent.repain@u-paris.fr
}

Morphological studies of the $\mathrm{C}_{60}$ growth on ferromagnetic metals, mainly by scanning tunneling microscopy (STM), show that this molecule hardly diffuses at room temperature [13] and even exhibits anomalous diffusion at higher temperature [14]. This generally leads to a rather disordered molecular layer at room temperature deposition [7]. Annealing to few hundreds of degrees above room temperature can give rise to a well-ordered molecular layer on Co [15] but induces a roughening of the interface, which is particularly clear on $\mathrm{Ni}(110)$ [16]. A possible explanation of this roughening is an interface reconstruction induced by the $\mathrm{C}_{60}$ and activated when the mobility is high enough.

Indeed, the tendency of $\mathrm{C}_{60}$ to generate a vacancy at its adsorption site has been demonstrated or suggested for various metal surfaces. An earlier photoemission study first reported the $\mathrm{C}_{60}$ bonding on $\mathrm{Al}(111)$ to be very strong, likely related to surface morphological changes [17]. Since then, diffraction studies have unambiguously demonstrated the presence of a single vacancy below each molecule of annealed $\mathrm{C}_{60}$ layers on $\operatorname{Pt}(111)$ [18], $\operatorname{Ag}(111)$ [19], and $\operatorname{Au}(111)$ [20]. Further STM [21] and molecular dynamics studies have shown that the vacancy formation during annealing was very gradual, probably due to the desorption of a residual $\mathrm{H}$ layer, and involving intermediate states with $\mathrm{C}_{60}$-adatom linkages [22].

An interesting question to address is how such an interface reconstruction can affect the interface properties. It has been noticed, for example, that the presence of vacancies reduced the interface melting temperature [21]. Concerning electronic properties, a pioneering work has shown that the annealing of ${ }_{a} \mathrm{C}_{60}$ layer on $\mathrm{Cu}(111)$ induces a nanohole made out of seven $\mathrm{Cu}$ vacancies below the molecules [23] concomitant with an enhanced charge transfer of three electrons per molecule [24]. On magnetic surfaces, such an interface reconstruction has never been clearly demonstrated to the best of our knowledge 
but more suggested to be the case on the basis of anomalous diffusion [14] or from the analysis of STM constrast [15,16]. More importantly, such a structural change of the interface can have a strong impact on the magnetic properties such as the interfacial magnetic anisotropy or the molecular spin polarization, as calculated in the case of Ni(111) [25] and $\mathrm{Cr}(001)$ [26].

In this paper, using grazing incidence x-ray diffraction (GIXD), we demonstrated that a $\mathrm{C}_{60}$ layer on $\mathrm{Co}(0001)$ indeed undergoes a gradual structural transition upon annealing to $650 \mathrm{~K}$, with the formation of a nanohole of seven vacancies below most of the molecules. Density functional theory (DFT) calculations show that such a configuration is lower in energy and leads to a strong modification of the interfacial magnetic anisotropy and of the $\mathrm{C}_{60}$ spin polarization and magnetization.

\section{EXPERIMENTAL RESULTS}

\section{A. Methods}

All the experiments have been performed on a $\operatorname{Co}(0001)$ single crystal that was cleaned under ultrahigh vacuum (UHV, base pressure in the $10^{-11}$ mbar range) by several subsequent cycles of sputtering with $\mathrm{Ar}^{+}$ions at $900 \mathrm{eV}$ and one hour annealing at $600 \mathrm{~K}$, i.e., below the martensitic transition [27]. The purity of the surface has been checked by Auger electron spectroscopy (AES) and STM (cf. Supplemental Material [28]). The $\mathrm{C}_{60}$ molecules were sublimated from a crucible heated by a homemade filament on the Co surface maintained at room temperature. The STM experiments were carried out on a VT-Omicron STM. Surface diffraction measurements were performed at the Surfaces interfaces x-ray Scattering (SixS) beamline at Synchrotron SOLEIL. In each UHV chamber, the $\mathrm{Co}(0001)$ is cleaned prior to $\mathrm{C}_{60}$ deposition and measurements are done during typically less than two days before preparing a new fresh sample. In the SixS UHV end station, a UHV preparation chamber equipped with low energy electron diffraction (LEED) and AES is connected to a UHV measurement chamber. The latter is coupled with a Z-axis diffractometer. Thanks to this setup, the transfer of the sample into the diffraction chamber is done at a high $10^{-11}$ mbar range, i.e., without any surface deterioration. As a summary, the measured samples have never experienced a pressure higher than $10^{-10}$ mbar. GIXD measurements were carried out at an energy of $18.41 \mathrm{keV}$ and an incident angle of $0.15^{\circ}$, with a photon flux around $10^{10}$ photons/s on the sample. We have checked (cf. Supplemental Material [28]) that the $\mathrm{C}_{60}$ layer is not degraded under such an $\mathrm{x}$-ray irradiation. Two detectors were used on two different samples to collect the scattered intensity: a point detector (entrance slits $5 \times 5 \mathrm{~mm}^{2}$, Fig. 3) and a 2D hybrid pixel detector (XPAD S140, Figs. 2 and 5). For the data acquired with the 2D detector, the BINoculars program [29] was used to process the whole data set and extract reciprocal space maps and crystal truncation rods (CTRs). The adequacy of the simulated CTRs intensities with the experimental data is quantified by the $\chi^{2}$ factor:

$$
\chi^{2}=\frac{1}{N_{\text {data }}-N_{p}} \sum_{i}^{N_{\text {data }}}\left|\frac{F_{i, \text { expt }}-F_{i, \text { th }}}{\sigma}\right|^{2},
$$

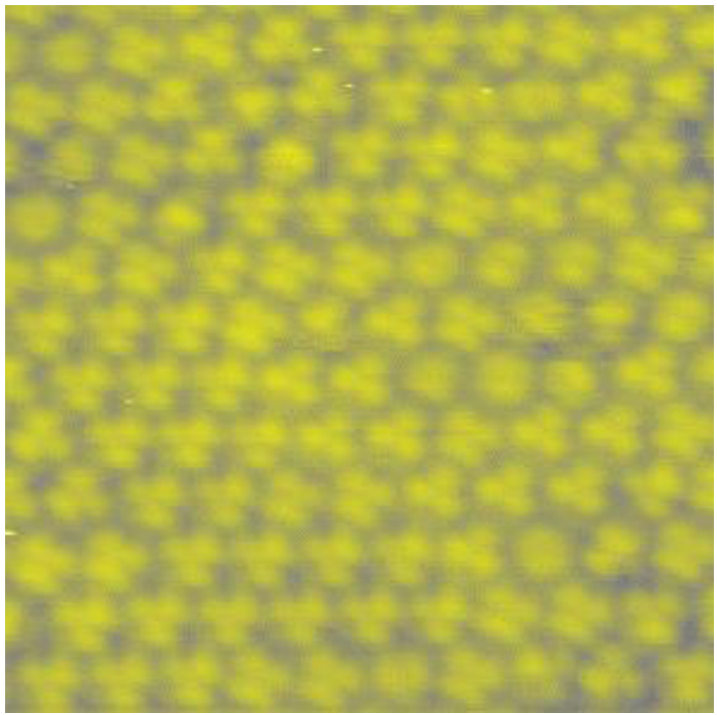

FIG. 1. $10 \times 10 \mathrm{~nm}^{2} \mathrm{STM}$ image of a $\mathrm{C}_{60}$ monolayer deposited at room temperature on $\operatorname{Co}(0001) . U_{t}=2 \mathrm{~V} ; I_{t}=1 \mathrm{nA}$.

where $N_{\text {data }}$ is the number of data points, $F_{\text {expt }}\left(F_{\text {th }}\right)$ the experimental factor structure modulus (simulated factor structure modulus), $N_{p}$ the number of fitting parameters, and $\sigma$ the error, which was estimated by the statistic integration error plus $10 \%$ of the factor structure modulus value to include the systematic error sources. We have indexed the $\mathrm{C}_{60}$ rods with reference to the $\operatorname{Co}(0001)$ hexagonal lattice [30]. The Miller indexes $H$ and $K$ refer to a reciprocal space vector lying in the plane of the sample surface and $L$ to the out-of-plane component.

\section{B. As deposited structure}

After deposition of around $1 \mathrm{ML}$ of $\mathrm{C}_{60}$ on the Co surface at room temperature one can observe by either LEED or GIXD a $4 \times 4$ superstructure with respect to the Co atomic lattice, as shown in the Supplemental Material (SM) [28]. It is worth noting that the use of a single crystal of $\mathrm{Co}$, although far from a real spintronic device, allows for obtaining a single orientation of molecular lattice, both at room temperature and after annealing, which is not the case on a Co thin film where a disordered structure is found at room temperature and several different domains are observed after annealing [15]. Figure 1 shows a small scale STM image of the $4 \times 4$ superstructure, with all the molecules at the same height on a perfectly ordered lattice. A closer inspection allows one to determine the adsorption configuration of the molecules, through the imaging of their lowest unoccupied molecular orbital (LUMO) levels $\left(U_{t}=2 \mathrm{~V}\right)$ [31]. A statistic done over more than 360 molecules on different images gives a proportion of $67 \pm 4 \%$ with a hexagonal adsorption with six different azimuthal orientations, $4 \pm 1 \%$ with a pentagonal adsorption, $7 \pm 1 \%$ with a $6-6$, and the rest undetermined.

The atomic structure of the $\mathrm{C}_{60} / \mathrm{Co}$ interface is probed by measuring the scattered intensity along the fractional rods related to the $4 \times 4$ superstructure. Due to the weak scattering factor of carbon and the diffuse scattering from the Co surface, we measured a reasonable signal to noise ratio only for $L$ 

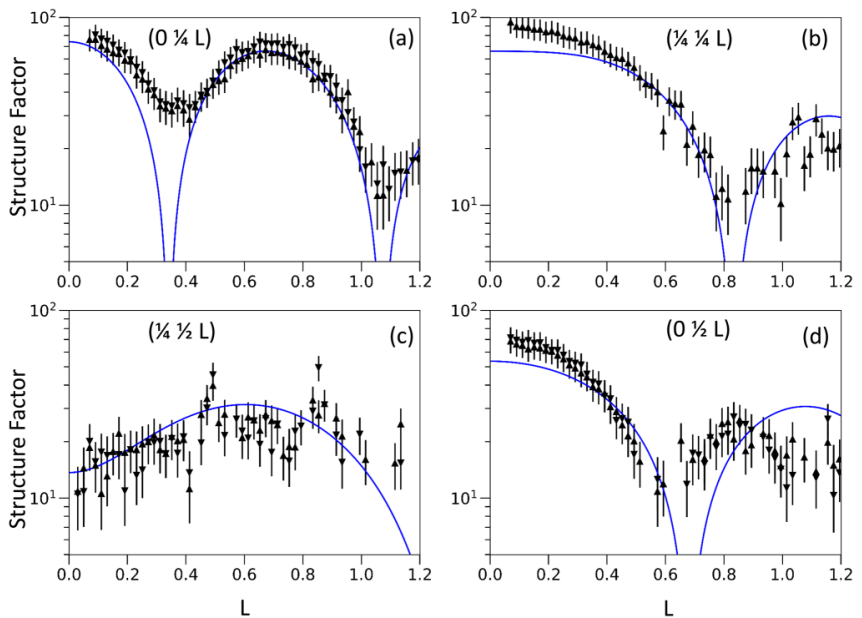

FIG. 2. Fractional rods of a $\mathrm{C}_{60}$ monolayer deposited at room temperature on $\mathrm{Co}(0001)$. (a) Up triangles: $(0$ 1/4 L ); down triangles: $(1 / 40 L)$. (b) Up triangles: $(1 / 41 / 4 L)$. (c) Up triangles: $(1 / 41 / 2$ $L$ ); down triangles: $(1 / 21 / 4 L)$. (d) Up triangles: $(01 / 2 L)$; down triangles $(1 / 20 L)$. The blue lines are the calculated structure factors for the molecules adsorbed on a hexagon.

below 1.2, as shown on Fig. 2 for seven different fractional rods. Considering mirror and threefold symmetries, we can gather those data in four different nonequivalent rods, the $(0$ $1 / 4 L)$ on Fig. $2(a)$, the $(01 / 2 L)$ on Fig. $2(b)$, the $(1 / 4$ $1 / 4 L)$ on Fig. $2(\mathrm{c})$, and the $(1 / 41 / 2 L)$ on Fig. 2(d). All the other diffraction orders show a vanishing intensity in our experimental conditions. Those rods are analyzed with a simple model, considering a hexagonal adsorption configuration for the molecules, as shown by STM, but with a random azimuthal orientation, averaging the structure factor over all the configurations. It is important to note that this model incorporates the above mentioned symmetries (cf. SM [28] for details). A good agreement with the data $\left(\chi^{2}=2.93\right)$ is obtained, as displayed by the blue curves in Fig. 2. A slightly better fit $\left(\chi^{2}=2.60\right.$; cf. SM [28]) is obtained by introducing the structure of the Co underlayer, with the $\mathrm{C}_{60}$ adsorbed with its center at $4.9 \AA$ above the surface [30] and the top Co atoms relaxed in the direction of the bulk by $0.22 \AA$, leading to a minimum distance between $\mathrm{C}$ and $\mathrm{Co}$ atoms ranging from 2.01 to $2.21 \AA$, depending on the azimuthal orientation. It is worth noting that an adsorption in hollow position gives a very close $\chi^{2}=2.64$ (cf. SM [28]) with minimum distances from 1.83 to $1.98 \AA$, in good agreement with DFT calculations (cf. Sec. III B). However, the tiny difference between the $\chi^{2}$ makes it difficult to ascribe unambiguously the favored adsorption site of $\mathrm{C}_{60}$ on Co from GIXD experiments.

As a conclusion of the STM and GIXD data, when deposited at room temperature on $\mathrm{Co}(0001)$, the $\mathrm{C}_{60}$ molecules arrange in a $4 \times 4$ regular lattice, with a favored hexagonal adsorption geometry and with a tendency of the Co atoms underneath the molecule to relax towards the bulk.

\section{Annealed structure}

As explained in the Introduction, $\mathrm{C}_{60}$ has a strong tendency to induce interface reconstructions on metals, with the creation of vacancies below the molecules. Most of the time,

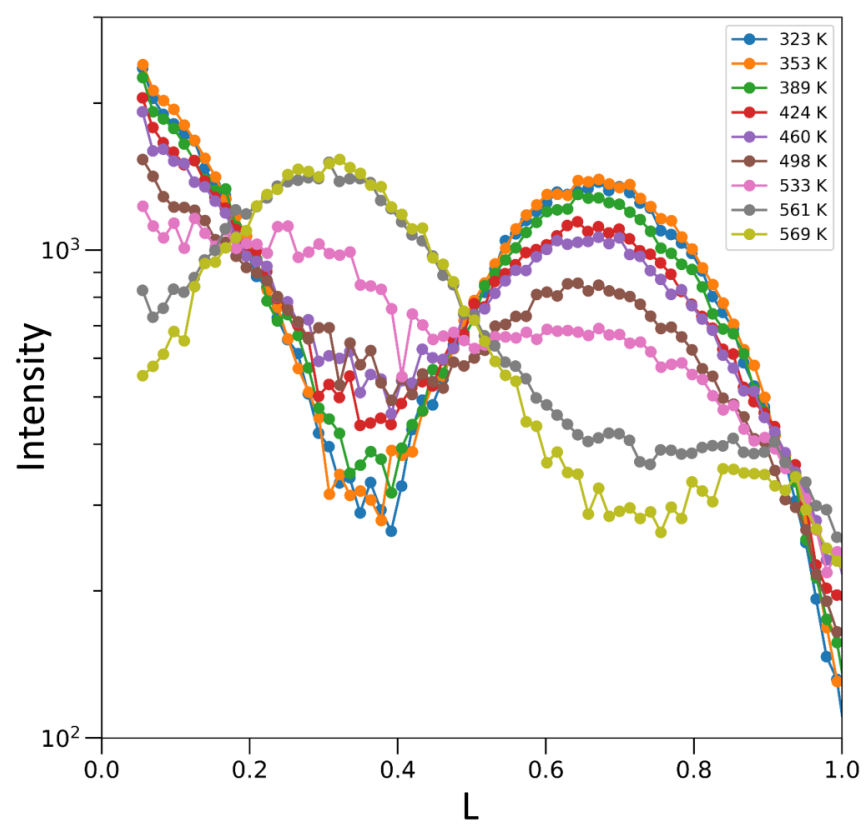

FIG. 3. $(1 / 40 L)$ fractional rod as function of temperature.

this structure is hindered at room temperature due to kinetic limitations. We have therefore decided to follow the structural change of the $\mathrm{C}_{60} / \mathrm{Co}$ interface in real time during a gradual annealing from room temperature to $570 \mathrm{~K}$. Figure 3 shows the evolution of the $(1 / 40 L)$ fractional rod for nine different temperatures between 323 and $569 \mathrm{~K}$. We observe a very gradual change in the intensity profile, starting at around $400 \mathrm{~K}$ and not yet fully completed at $570 \mathrm{~K}$. Finally, the overall curve is drastically modified between room temperature and $570 \mathrm{~K}$ with minima that change to maxima and vice versa. When going back to room temperature, the intensity profile is found to be irreversibly modified by the annealing, with no significant difference with the $570 \mathrm{~K}$ measurement. Such a strong modification of the scattered intensity is obviously related to a large structural change of the $\mathrm{C}_{60} / \mathrm{Co}$ interface.

In order to determine this new structure, we have first performed STM imaging of a similarly annealed sample. Large scale images show a surface roughening with a decrease of the Co terrace sizes. At smaller scale (cf. Fig. 4), one can observe that all the $\mathrm{C}_{60}$ molecules are not in the same plane, the difference in height between top (yellow) and bottom (blue) molecules being $1.3 \pm 0.6 \AA$, i.e., lower than a Co step edge $(2.035 \AA)$. The study of the adsorption geometries gives a very different result for top and bottom molecules. The ones in the top configuration have adsorption geometries rather similar to what was found after the room temperature deposition (73 $\pm 5 \%$ in hexagonal, $21 \pm 3 \%$ in pentagonal, $2 \pm 1 \%$ in $6-6$, and $3 \%$ undetermined over 341 molecules), while the ones in the bottom configuration have a tendency to switch to a pentagonal adsorption geometry $(11 \pm 3 \%$ in hexagonal, $53 \pm 6 \%$ in pentagonal, $4 \pm 2 \%$ in $6-6$, and $32 \pm$ $5 \%$ undetermined over 133 molecules). The $1.3 \AA$ difference in height between bottom and top $\mathrm{C}_{60}$ is larger than what one can expect from a purely electronic effect coming from different adsorption geometries, especially at a gap voltage of $2 \mathrm{~V}$ that a priori integrates the molecular LUMO level 


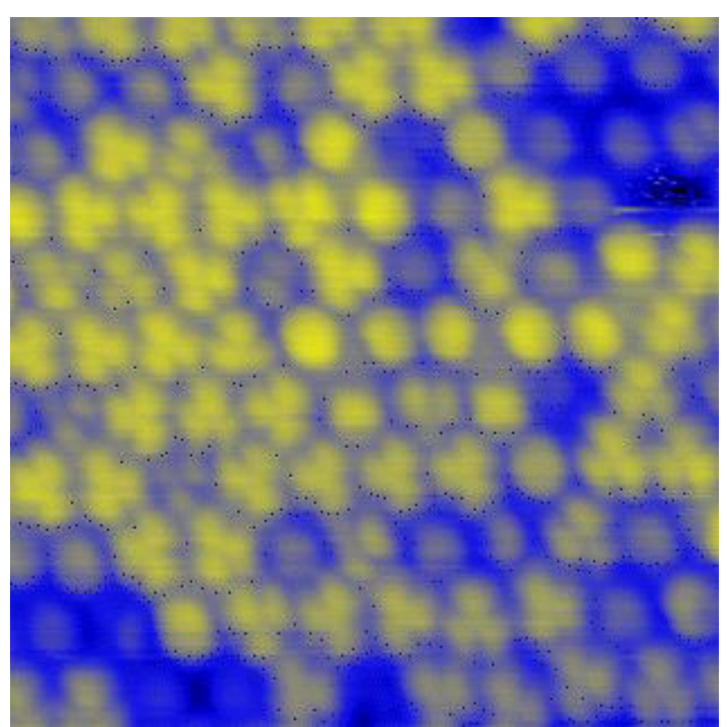

FIG. 4. $10 \times 10 \mathrm{~nm}^{2}$ STM image of a $\mathrm{C}_{60}$ monolayer on $\mathrm{Co}(0001)$ after annealing at $570 \mathrm{~K} . U_{t}=2 \mathrm{~V} ; I_{t}=1 \mathrm{nA}$.

for all adsorption geometries. Moreover, similar adsorption geometries are observed in top and bottom configurations. It is therefore likely that the bottom configuration is induced by a structural change, i.e., cobalt vacancies under the molecules. Possibly, the Co atoms ejected to create the vacancies could diffuse at the interface to create new Co terraces.

STM is unable to go further in the structural determination of the interface. We have therefore recorded the structure factor of fractional rods by GIXD, on a sample that was annealed at $660 \mathrm{~K}$ (30 min ramp from room temperature to $660 \mathrm{~K}$ followed by $15 \mathrm{~min}$ at $660 \mathrm{~K}$ ) but measured at room temperature. The choice of the annealing temperature was dictated by the previous observations that $570 \mathrm{~K}$ provided only a partial transformation of the interfacial structure and by the limitation due to the martensitic transition (hexagonal close-packed to face-centered cubic structure at $693 \mathrm{~K}$, that we already partially observe by diffraction at our $660 \mathrm{~K}$ annealing temperature). Figure 5 shows the same seven fractional rods as measured in Fig. 2. The first observation is that the signal to noise ratio is generally lower, which limits our measurements to a maximum $L$ value of 0.8 instead of 1.2. This can be due in part to the smaller size of coherent $\mathrm{C}_{60}$ crystals, due to the random mixing between bottom and top configurations observed by STM and in another part to the roughening of the Co surface that increases the diffuse scattering. In order to reproduce the measured structure factors, we have compared our data with models including from one to seven Co vacancies below the $\mathrm{C}_{60}$ molecules, with the buckyballs adsorbed on a pentagonal geometry and an averaging over all the possible azimuthal orientations. Once again, our models respect the measured symmetries (cf. SM [28] for details). The seven vacancies model fits the data reasonably well $\left(\chi^{2}=2.61\right.$ instead of $8.74,4.34$, and 4.04 respectively for the one, three, and six vacancies; cf. SM [28] for more details). We find that the $\mathrm{C}_{60}$ molecules partially fill those vacancies islands, getting close to the newly created surface, with a minimum distance between $\mathrm{C}$ and $\mathrm{Co}$ atoms ranging from 1.65 to $1.69 \AA$. It is worth
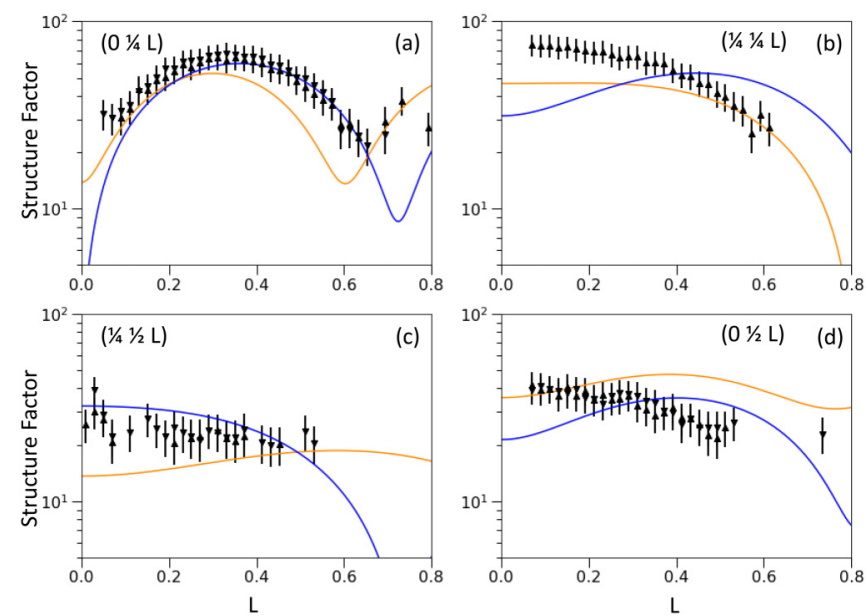

FIG. 5. Fractional rods of a $\mathrm{C}_{60}$ monolayer on $\mathrm{Co}(0001)$ after annealing at $660 \mathrm{~K}$ for $15 \mathrm{~min}$. (a) Up triangles: $(01 / 4 \mathrm{~L})$; down triangles: $(1 / 40 L)$. (b) Up triangles: $(1 / 41 / 4 L)$. (c) Up triangles: $(1 / 41 / 2 L)$; down triangles: $(1 / 21 / 4 L)$. (d) Up triangles: $(01 / 2 L)$; down triangles $(1 / 20 L)$. The blue (orange) lines are the calculated structure factor for the molecules adsorbed on a pentagon, nested in a seven (six) vacancies hole.

noting that the comparison between the data and the model is not perfect for the $(01 / 2 L)$ and $(1 / 41 / 4 L)$ rods. The agreement can be slightly improved $\left(\chi^{2}=1.54\right)$ by taking into account a mixing between the annealed (80\%) and nonannealed models (20\%). We have also not considered a possible deformation of the molecule (as discussed in Sec. III B) and displacements of the Co atoms, as it would require too many fitting parameters as compared to our data set. Finally, note that a mix of seven and six Co vacancies models (cf. blue and orange curves in Fig. 5 for both fits) is also a possible way to improve the fit, although the six vacancies model is found to have a much larger formation energy (cf. Sec. III C).

To conclude the analysis of the STM and GIXD data after an annealing at $660 \mathrm{~K}$, we find that the $\mathrm{C}_{60}$ molecules stay in a $4 \times 4$ regular lattice, with most of the molecules having a seven cobalt vacancies hole below, acting like a nanonest for the molecule. As shown in the following, this finding is supported by DFT calculations that also demonstrate how this change of interfacial structure can strongly affect the magnetic and spin transport properties. We can also note that such an interface reconstruction has already been suggested on the basis of STM images for annealed $\mathrm{C}_{60}$ molecules deposited on a Co film grown on $\mathrm{Au}(111)$ [15]. However, in the latter case, several orientations of the $\mathrm{C}_{60}$ two-dimensional crystals are observed and this reconstruction coexists with other structures.

\section{THEORETICAL STUDY}

We present in this section theoretical results based on DFT calculations to confirm the increased stability of the seven vacancies model and to make some further predictions on the physical properties. We have studied three different $\mathrm{C}_{60}-\mathrm{Co}$ reconstructed surfaces compared to the flat $\mathrm{Co}(0001)$ surface. 


\section{A. Methodology}

We have performed DFT calculations using the generalized gradient approximation (GGA) in the Perdew, Berke, and Ernzherof (PBE [32]) parametrization based on plane waves expansion of the valence electrons wave functions: QUANTUM ESPRESSO (QE) [33,34]. We have used ultrasoft pseudopotentials [35] and the size of the basis, controlled by the energy cutoff, was taken equal to $30 \mathrm{Ry}$ and $300 \mathrm{Ry}$ for the wave function and the charge density, respectively. Marzari-Vanderbilt cold smearing with a broadening of $0.05 \mathrm{eV}$ has been used. Site and orbital resolved quantities are obtained by projection of the wave functions onto orthogonalized atomic pseudowave functions in a Lowdin manner, while vacuum density of states is obtained by integrating the electronic density in a small $0.5 \AA^{3}$ box above the deposited $\mathrm{C}_{60}$ molecule.

For the calculation of the magnetocrystalline anisotropy (MCA) we have used fully relativistic pseudopotentials [36] and applied the magnetic force theorem [37-40] (FT) to evaluate the MCA and its local components [7]. This approach is based on a three step process [38]: (i) a self-consistent (scf) calculation with a scalar relativistic pseudopotential (without spin-orbit coupling), followed by (ii) a non-self-consistent (nscf) calculation with a fully relativistic pseudopotential including spin-orbit coupling starting from the scf electron density rotated to the specified magnetization orientations. Finally, (iii) the MCA is obtained from the variation of the band energy term and its site and orbital decomposed components from the projected quantities. In all calculations, the scf loop is performed with $5 \times 5 k$ points of the two dimensional Brillouin zone, while the nscf calculations are performed with a denser sampling of $7 \times 7 k$ points in the full Brillouin zone.

\section{B. Geometric structures}

We have considered several possible geometric configurations according to the experiment: a $\mathrm{C}_{60}$ molecule deposited on a bare Co hexagonal close packed (hcp) (0001) surface and on three, six, and seven surface vacancies represented in Fig. 6. The hcp (0001) slab is composed of five layers of $4 \times 4$ Co atoms. To reproduce the nest, three, six, and seven atoms are removed from the outermost layer and the structures are relaxed with $\mathrm{QE}$. The $\mathrm{C}_{60}$ is then added on its hexagonal facet and the structure is relaxed again. Note that, in a previous work [41], we investigated different adsorption geometries of $\mathrm{C}_{60}$ on the flat $\mathrm{Co}$ and found that the so-called 5-6 bond and hexagonal adsorption geometries are the most stable. In this work we have also considered the hexagonal geometry but, different from Ref. [41] where the hexagon was centered on a surface Co atom, here one-half of the carbon atom of the hexagonal facet is on top of Co. This corresponds locally to the adsorption geometry adopted by a graphene sheet on cobalt [42]. Interestingly, the relaxed structures show rather different features. On the bare surface the adsorbed molecule is almost the same as the free molecule but the Co atoms underneath the $\mathrm{C}$ relax towards the bulk by $0.1 \AA$, in good agreement with the experimental finding. In the case of the three vacancies the adsorbed molecule gets tilted and adopts a vertex geometry where the vertex is above the three vacancies hole and all its neighboring atoms (plus some of its second neighbors) hybridize with the atoms of the cobalt surface.
Side view

(a)

(b)
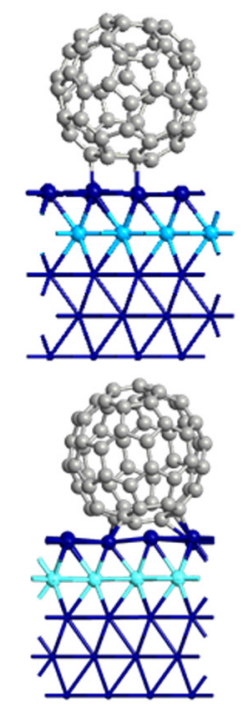

(c)

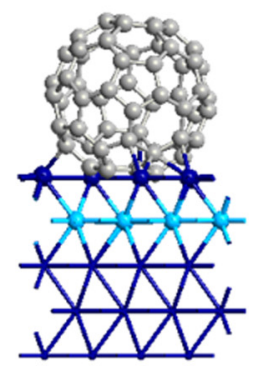

(d)

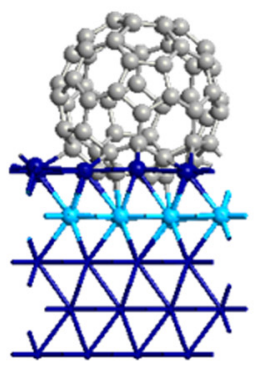

Top view
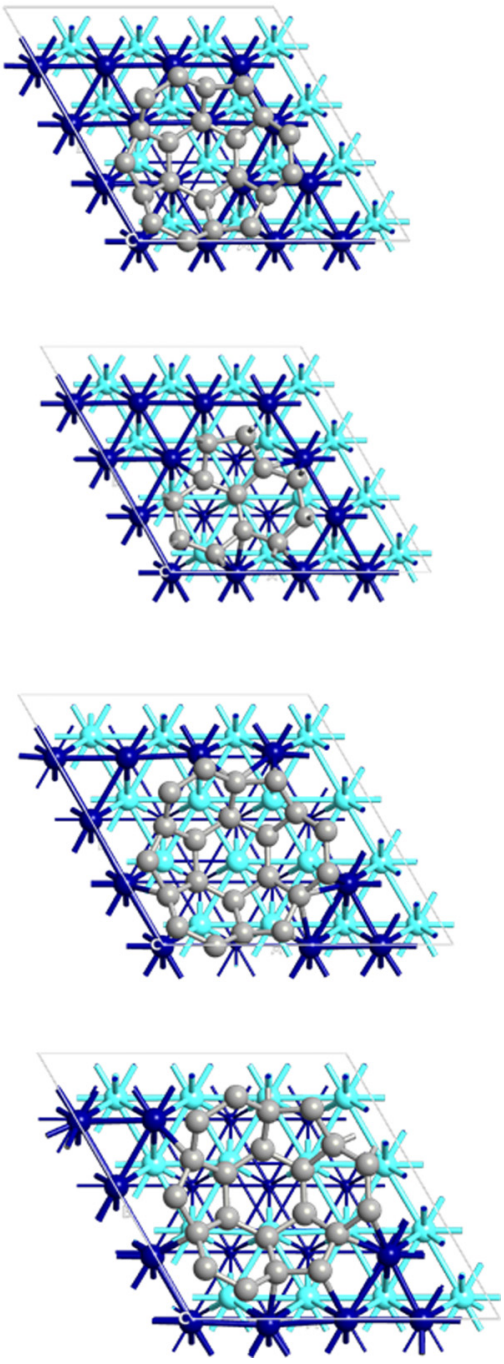

FIG. 6. Side and top views of the structures considered: $\mathrm{C}_{60}$ on (a) a flat $\operatorname{Co}(0001)$ surface and on the reconstructed surface of (b) three vacancies, (c) six vacancies, and (d) seven vacancies. Carbon atoms are shown in gray, surface Co atoms in dark blue, sublayer Co atoms in light blue, and deeper layers in dark blue with a smaller radius (and smaller bonds). For (a), (c), and (d) top views we have shown only the hexagonal facet and its three neighboring hexagonal and pentagonal facets. For (b) (three vacancies structure) that adopts a vertex geometry we have shown the vertex with two hexagonal and one pentagonal facets.

On the six vacancies the adsorbed molecule is almost not deformed and the binding to the cobalt surface mainly occurs via a small number of carbon atoms neighboring the hexagonal facet, while the atoms of the hexagonal facet remain far from the sublayer. In short the buckyball does not fall into the triangular hole. In contrast, the buckyball penetrates into the hexagonal hole of the seven vacancies, many bonds are formed with the Co atoms, and the $\mathrm{C}_{60}$ molecule is flattened by almost $0.1 \AA$ (distance between the two hexagonal 
TABLE I. Computed adsorption energies $E_{\text {ad }}$, vacancy formation energies $E_{\mathrm{vac}}$, and adsorption energies corrected for vacancy formation energies $E_{\text {ad }}+E_{\text {vac }}$ for the various systems considered.

\begin{tabular}{lcrrr}
\hline \hline Vacancies & 0 & 3 & \multicolumn{1}{c}{6} & \multicolumn{1}{c}{7} \\
\hline$E_{\text {ad }}(\mathrm{eV})$ & -1.16 & -3.77 & -0.58 & -4.61 \\
$E_{\text {vac }}(\mathrm{eV})$ & 0 & 1.82 & 2.68 & 2.42 \\
$E_{\text {ad }}+E_{\text {vac }}(\mathrm{eV})$ & -1.16 & -1.95 & 2.10 & -2.19 \\
\hline \hline
\end{tabular}

facets). Note that the geometry obtained for $\mathrm{C}_{60}$ in the sevenvacancies hole is very similar to the one described in Ref. [23] for $\mathrm{C}_{60}$ on the seven-vacancies reconstruction of $\mathrm{Cu}(111)$. These specific characteristics will reflect on the energetic of the adsorption as shown in the next section. Finally, one can note that the structural configurations which are found by DFT do not strictly respect the mirror and sixfold symmetries observed experimentally. Therefore, the calculated $\mathrm{C}_{60}$ and Co relaxations cannot be used directly to calculate a structure factor that could be compared with experimental data. An averaging over different configurations could allow one to recover such symmetries but is out of the scope of this paper.

\section{Energetics}

Using QE we are able to extract the total energy of the various systems. To evaluate the relative stability of the different systems we have followed the same methodology as in Ref. [25]. The creation process can be decomposed into two steps: (i) $N(N=3,6,7)$ Co atoms are extracted from the surface layer of the flat Co slab (vacancy formation energy $E_{\mathrm{vac}}$ ); then (ii) the $\mathrm{C}_{60}$ is adsorbed in the created hole (adsorption energy $\left.E_{\text {ad }}\right)$. Finally, the creation energy is the summation of both the vacancy formation and the adsorption energies.

The vacancy formation energy $E_{\mathrm{vac}}$ is obtained by the difference between the energy of the reconstructed $N$-vacancies Co slab $E_{\text {Nvac }}^{\text {tot }}$ and the flat slab $E_{\text {slab }}^{\text {tot }}$ corrected by the energy of the $N$ Co atom $N \times E_{\text {atom }}$ extracted from the surface:

$$
E_{\mathrm{vac}}=E_{\mathrm{Nvac}}^{\mathrm{tot}}+N \times E_{\mathrm{atom}}-E_{\text {slab }}^{\mathrm{tot}} .
$$

The computation of $E_{\text {atom }}$ depends on the mechanism involved in the extraction process. It is quite improbable that the extracted atom goes into the gas phase and most likely the atom will diffuse on the surface and might eventually stick at a kinked step or else diffuse in the bulk. Since the calculation of atom kink energy can be delicate and Pang et al. in Ref. [25] showed that it does not change the result with respect to the stability of the process for a Ni surface, we have used the bulk energy for $E_{\text {atom }}$.

The adsorption energy $E_{\text {ad }}$ is more directly obtained by the difference between the total energy of the $\mathrm{C}_{60}$ in contact with the $N$-vacancies Co slab $E_{\text {Nvac-C } 60}$ and the two separate isolated systems:

$$
E_{\mathrm{ad}}=E_{\mathrm{Nvac}-\mathrm{C}_{60}}-E_{\mathrm{C}_{60}}-E_{\mathrm{Nvac}} .
$$

The adsorption energies corrected for the vacancy formation energies $E_{\mathrm{ad}}+E_{\mathrm{vac}}$ have been computed and are listed in Table I.

According to Table I, the most stable system is the sevenvacancies model. The adsorption energy on the flat surface
$(1.16 \mathrm{eV})$ is relatively small in agreement with our previous findings in Ref. [41]. The adsorption geometry is a priori not the most stable but very close to the other hexagonal geometry studied in Ref. [41] and discussed in our previous section. The three-vacancies system is more stable than the flat surface due to its strong adsorption energy, more than three times larger than on the flat surface that compensates the extraction of the three atoms. The six-vacancies system is unstable because of a very small adsorption energy which is due to the peculiar adsorption geometry of the $\mathrm{C}_{60}$ where the hexagonal facet weakly binds to the substrate. Indeed none of the atoms of the hexagonal facet have a distance shorter than $2.12 \AA$ and the binding occurs via a few side atoms. On the contrary, there is a very strong binding of the $\mathrm{C}_{60}$ to the seven-vacancies hole, which makes this configuration the most stable, in very good agreement with the experimental finding of Sec. II C. Hence, in the following, we will only consider the seven-vacancies system and compare it with the flat surface case.

\section{Magnetic properties}

We report in this section magnetic properties of the $\mathrm{C}_{60}-\mathrm{Co}$ seven-vacancies system compared to the flat surface. We will focus on two physical properties: the tunneling magnetoresistance and the magnetocrystalline anisotropy energy.

\section{Tunneling magnetoresistance (TMR)}

Let us first investigate theoretically how the TMR is modified when the system adopts its reconstructed structure. In a simplified Tersoff-Hamann approach [43,44], the spin-polarized differential conductance is related to the spinresolved local density of states (LDOS) of the sample at the STM tip position [41]:

$$
G=\frac{d I}{d V} \propto \sum_{\sigma} n_{\mathrm{T}}^{\sigma} n_{\mathrm{S}}^{\sigma}\left(R_{\mathrm{T}}, E_{F}+e V\right),
$$

where $n_{\mathrm{T}}^{\sigma}, n_{\mathrm{S}}^{\sigma}\left(R_{\mathrm{T}}, E_{F}+e V\right)$ are, respectively, the spindependent tip DOS (assumed to be constant in energy but spin dependent if the tip is magnetic) and vacuum LDOS of the sample $\left(\mathrm{C}_{60}\right.$ molecule deposited on surface $)$ calculated at the tip position $R_{\mathrm{T}}$ above the molecule and at the energy corresponding to applied voltage $V$. The TMR is then defined as $\left(n_{\mathrm{S}}^{\uparrow}-n_{\mathrm{S}}^{\downarrow}\right) /\left(n_{\mathrm{S}}^{\uparrow}+n_{\mathrm{S}}^{\downarrow}\right)$

In Fig. 7 the vacuum density of states (VDOS) in a box of $0.5 \AA^{3}$ at $4 \AA$ upon $\mathrm{C}_{60}$ for up and down spins is presented. The VDOS shows clear differences whether the molecule is on the flat or on the reconstructed surface. In both cases, there exist peaks at approximately the same energies corresponding to the position of HOMO, LUMO, and LUMO+1 states of the molecule; however, the amplitudes of the peaks are not the same. In particular, the HOMO states at $-1.5 \mathrm{eV}$ below the Fermi level are much more visible in the case of the seven vacancies. In addition, the difference between the spin up and down components is more pronounced for the reconstructed surface. This indicates a possibly large tunneling magnetoresistance in particular above the Fermi level dominated by the LUMO states. Indeed, in the energy range of $[0.2,0.6] \mathrm{eV}$ a large $40 \%$ positive TMR is observed. In the case of the flat surface large TMR are also observed but on smaller energy 

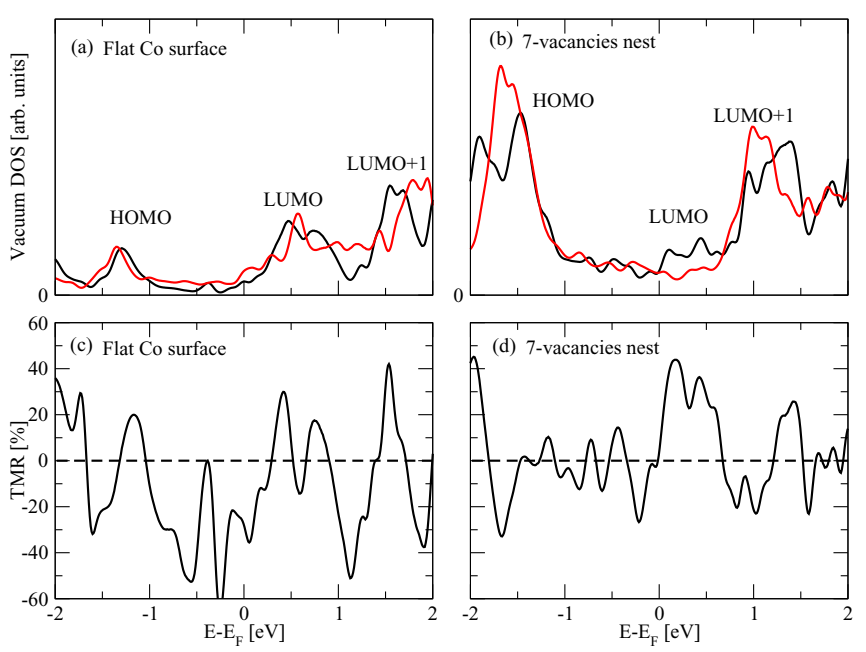

FIG. 7. Calculated vacuum density of states in a box of $0.5 \times$ $0.5 \times 0.5 \AA^{3}$ at $4 \AA$ above $\mathrm{C}_{60}$ (a) for the flat and (b) for the reconstructed seven-vacancies structure. Spins up and down are represented in black and red, respectively. (c) Tunnel magnetoresistance above the $\mathrm{C}_{60}$ on a flat surface. (d) Tunnel magnetoresistance above the $\mathrm{C}_{60}$ on the reconstructed seven-vacancies structure.

ranges and essentially in zones of low electronic densities where the experimental signal to noise should be smaller. Finally, it is worth mentioning that the VDOS of the $\mathrm{C}_{60}$ on the flat surface differs significantly from the one shown in Ref. [41] for the hexagonal adsorption geometry and our VDOS resembles more the one obtained for the so-called 5:6 bond geometry. This is due to the different hexagonal adsorption geometry chosen in Ref. [41], where the hexagon is centered on a Co surface atom (top), while here it is centered on a hollow site. Our geometry is therefore closer to the 5:6 bond one of Ref. [41].

\section{Magnetocrystalline anisotropy energy}

Let us first note that the MCA is a very delicate quantity to calculate and one should always be cautious about the real significance of quantitative values and rather focus on general trends. We have calculated the site and orbital decomposed MCA (defined as the energy difference between in-plane and out-of-plane magnetization) for $\mathrm{C}_{60}$ adsorbed on the flat and reconstructed seven-vacancies Co surfaces. Here positive/negative MCA values indicate out-of-plane/in-plane anisotropy, respectively. The results are shown in Fig. 8. As already pointed out in our previous publication [7] the dominant effect of the $\mathrm{C}_{60}$ adsorption on the flat Co surface is driven by the hybridization between carbon $p_{z, \mathrm{C}}$ orbital and cobalt $d_{z^{2} \text {, Co }}$ (and $d_{x z}-d_{y z}$ ). This hybridization tends to cancel the associated MCA component of the surface Co atoms in direct contact with the molecule and hence favors an out-of-plane magnetization since $d_{z^{2}}$ and $d_{x z}-d_{y z}$ contributions are both negative (red and blue curves in Fig. 8). The case of the reconstructed seven vacancies is more complex due to its geometry where carbon atoms hybridize both with the surface and subsurface Co atoms. While the hybridization with the sublayer still preferentially involves $p_{z, \mathrm{C}}$ of the hexagonal facet and $d_{z^{2} \text {,Co }}$ of the sublayer, similar to the flat surface, it
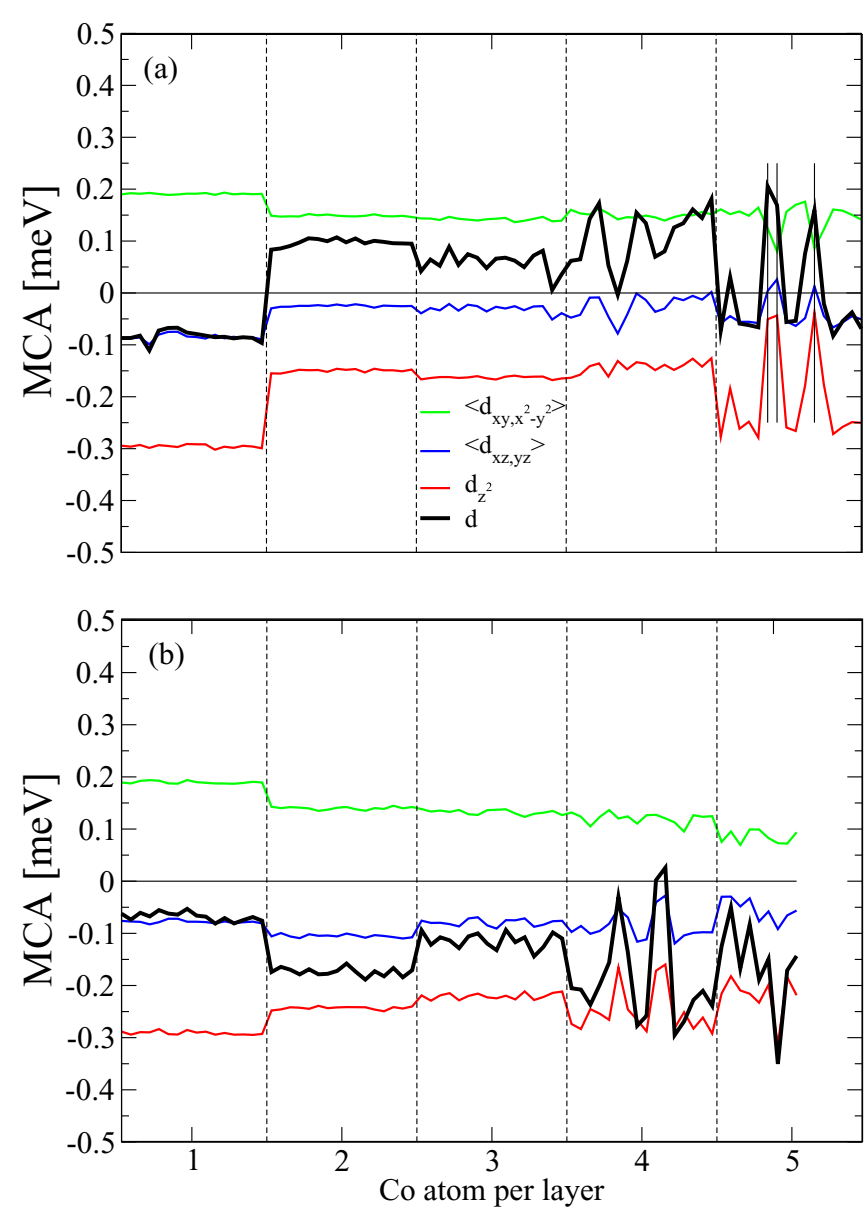

FIG. 8. Site and orbital decomposed $\mathrm{MCA}=E_{\|}-E_{\perp}$ of $\mathrm{C}_{60}$ deposited (a) on a flat and (b) on a reconstructed seven-vacancies structure. The $\mathrm{C}_{60}$ is deposited on the fifth layer. Each Co layer contains $4 \times 4=16 \mathrm{Co}$ atoms except the fifth layer of the reconstructed surface that contains only nine atoms. Vertical dotted lines show the separation between each Co layer. Vertical full lines indicate the position of the Co atoms with the shortest distance to $\mathrm{C}_{60}$ in the case of the flat surface.

is no longer the case for the surface layer where basically all orbitals are involved. As a result the MCA of all orbital components of the surface layer are affected and tend to cancel out. The directional character leading to a reinforcement of the out-of-plane anisotropy is therefore lost. The sublayer still behaves very much like the surface layer of the flat surface. Finally, one can note a global downward shift (towards negative values) of the $d_{z^{2}}$ (and to a lesser extent the $d_{x z}-d_{y z}$ ) component of the MCA for the reconstructed surface. This is attributed to relaxation effects, in particular different interlayer distances between the flat and the reconstructed surface. This behavior is probably partly artificial, due to the limited number of Co layers, and illustrates the difficulty in obtaining quantitative evaluation of the MCA. In conclusion, we confirm the general trend of reinforcement of the out-of-plane MCA by adsorption of $\mathrm{C}_{60}$ on the flat Co surface, induced by the directional $p_{z, \mathrm{C}}-d_{z^{2} \text {, Co }}$ hybridization. In contrast, the more complex geometry of the reconstructed surface blurs out the 
orbital directionality and we expect a smaller effect than the one observed for the flat surface.

\section{CONCLUSION}

We have studied the structural modification induced at the interface between a Co surface and a $\mathrm{C}_{60}$ layer by annealing above $550 \mathrm{~K}$. Both scanning tunneling microscopy and grazing incidence $\mathrm{x}$-ray diffraction show that the buckyballs generate a rearrangement of the Co atoms at the interface layer. At room temperature, the molecules adsorb every $4 \times 4$ Co atomic site on the flat Co surface while, after annealing, each molecule induces the creation of a small nest of seven Co vacancies in which the molecule self-inserts. Density functional theory results show that this annealed interfacial structure increases the number of $\mathrm{Co}-\mathrm{C}$ bonds, which is energetically favorable. Moreover, we have calculated that such a structural change has a strong impact on the magnetic properties of this new spinterface. We find that the out-of-plane magnetic anisotropy that was induced by the C $p_{z}-\mathrm{Co} d_{z^{2}}$ hybridization is weakened by the roughening of the interface. In contrast, the molecular spin polarization is enhanced at the energy of the molecular levels (both in negative and positive polarities), expecting larger magnetoresistance in such annealed samples. Although this study has been performed on a model system consisting of a molecular monolayer deposited on a single crystal, we think that our observation of an interfacial reconstruction upon annealing is quite general, even to more disordered systems, and that those results can be of direct interest for the realization and the understanding of more efficient $\mathrm{C}_{60}$-based spintronic devices. More generally, it shows that molecule/ferromagnet interfaces can be structurally more complex than expected, with magnetic properties highly sensitive to the structure of the interface.

\section{ACKNOWLEDGMENTS}

This project has received funding from the European Union's Horizon 2020 research and innovation programme under Grant Agreement No. [766726]. We thank L. Kipgen for proofreading the manuscript.
[1] C. Barraud, P. Seneor, R. Mattana, S. Fusil, K. Bouzehouane, C. Deranlot, P. Graziosi, L. Hueso, I. Bergenti, V. Dediu, F. Petroff, and A. Fert, Nat. Phys. 6, 615 (2010).

[2] K. V. Raman, A. M. Kamerbeek, A. Mukherjee, N. Atodiresei, T. K. Sen, P. Lazić, V. Caciuc, R. Michel, D. Stalke, S. K. Mandal, S. Blügel, M. Münzenberg, and J. S. Moodera, Nature (London) 493, 509 (2013).

[3] M. Gobbi, F. Golmar, R. Llopis, F. Casanova, and L. E. Hueso, Adv. Mater. 23, 1609 (2011).

[4] X. Zhang, S. Mizukami, T. Kubota, Q. Ma, M. Oogane, H. Naganuma, Y. Ando, and T. Miyazaki, Nat. Commun. 4, 1392 (2013).

[5] R. Lin, F. Wang, M. Wohlgenannt, C. He, X. Zhai, and Y. Suzuki, Synth. Met. 161, 553 (2011), The 3rd International Meeting on Spins in Organic Semiconductors 2010 (SPINOS III).

[6] M. Galbiati, S. Tatay, C. Barraud, A. V. Dediu, F. Petroff, R. Mattana, and P. Seneor, MRS Bull. 39, 602 (2014).

[7] K. Bairagi, A. Bellec, V. Repain, C. Chacon, Y. Girard, Y. Garreau, J. Lagoute, S. Rousset, R. Breitwieser, Y.-C. Hu, Y. C. Chao, W. W. Pai, D. Li, A. Smogunov, and C. Barreteau, Phys. Rev. Lett. 114, 247203 (2015).

[8] T. Moorsom, S. Alghamdi, S. Stansill, E. Poli, G. Teobaldi, M. Beg, H. Fangohr, M. Rogers, Z. Aslam, M. Ali, B. J. Hickey, and O. Cespedes, Phys. Rev. B 101, 060408(R) (2020)

[9] K. Bairagi, A. Bellec, V. Repain, C. Fourmental, C. Chacon, Y. Girard, J. Lagoute, S. Rousset, L. Le Laurent, A. Smogunov, and C. Barreteau, Phys. Rev. B 98, 085432 (2018).

[10] S. Mallik, P. Sharangi, B. Sahoo, S. Mattauch, T. Brückel, and S. Bedanta, Appl. Phys. Lett. 115, 242405 (2019).

[11] D.-O. Kim, J. W. Choi, and D. R. Lee, J. Magn. Magn. Mater. 401, 506 (2016).

[12] K. V. Raman, S. M. Watson, J. H. Shim, J. A. Borchers, J. Chang, and J. S. Moodera, Phys. Rev. B 80, 195212 (2009).
[13] M. Cummings, S. Gliga, B. Lukanov, E. I. Altman, M. Bode, and E. V. Barrera, Surf. Sci. 605, 72 (2011).

[14] C. H. Lin, K. C. Lin, T. B. Tang, and W. W. Pai, J. Nanosci. Nanotechnol. 8, 602 (2008).

[15] J. Kollamana, Z. Wei, M. Laux, J. Stöckl, B. Stadtmüller, M. Cinchetti, and M. Aeschlimann, J. Phys. Chem. C 120, 7568 (2016).

[16] P. W. Murray, M. Ø. Pedersen, E. Lægsgaard, I. Stensgaard, and F. Besenbacher, Phys. Rev. B 55, 9360 (1997).

[17] A. J. Maxwell, P. A. Brühwiler, S. Andersson, D. Arvanitis, B. Hernnäs, O. Karis, D. C. Mancini, N. Mårtensson, S. M. Gray, M. K. -J. Johansson, and L. S. O. Johansson, Phys. Rev. B 52, R5546 (1995).

[18] R. Felici, M. Pedio, F. Borgatti, S. Iannotta, M. Capozi, G. Ciullo, and A. Stierle, Nat. Mater. 4, 688 (2005).

[19] H. I. Li, K. Pussi, K. J. Hanna, L.-L. Wang, D. D. Johnson, H.P. Cheng, H. Shin, S. Curtarolo, W. Moritz, J. A. Smerdon, R. McGrath, and R. D. Diehl, Phys. Rev. Lett. 103, 056101 (2009).

[20] X. Torrelles, M. Pedio, C. Cepek, and R. Felici, Phys. Rev. B 86, 075461 (2012).

[21] H. Shin, A. Schwarze, R. D. Diehl, K. Pussi, A. Colombier, É. Gaudry, J. Ledieu, G. M. McGuirk, L. N. Serkovic Loli, V. Fournée, L. L. Wang, G. Schull, and R. Berndt, Phys. Rev. B 89, 245428 (2014).

[22] A. L. Pinardi, G. Biddau, K. v. D. Ruit, G. Otero-Irurueta, S. Gardonio, S. Lizzit, R. Schennach, C. F. J. Flipse, M. F. López, J. Méndez, R. Pérez, and J. A. Martín-Gago, Nanotechnology 25, 385602 (2014).

[23] G. Xu, X.-Q. Shi, R. Q. Zhang, W. W. Pai, H. T. Jeng, and M. A. Van Hove, Phys. Rev. B 86, 075419 (2012).

[24] W. W. Pai, H. T. Jeng, C.-M. Cheng, C.-H. Lin, X. Xiao, A. Zhao, X. Zhang, G. Xu, X. Q. Shi, M. A. Van Hove, C.-S. Hsue, and K.-D. Tsuei, Phys. Rev. Lett. 104, 036103 (2010).

[25] R. Pang, X. Shi, and M. A. Van Hove, J. Am. Chem. Soc. 138, 4029 (2016). 
[26] Y. Shao, R. Pang, H. Pan, and X. Shi, J. Chem. Phys. 148, 114704 (2018).

[27] C. R. Houska, B. L. Averbach, and M. Cohen, Acta Metall. 8, 81 (1960).

[28] See Supplemental Material at http://link.aps.org/supplemental/ 10.1103/PhysRevB.104.235413 for further details of the experimental characterization of the sample and the different models used to fit the x-ray diffraction data, and some simulations of the molecular diffusion factor in different geometries to support some conclusions of the main text.

[29] S. Roobol, W. Onderwaater, J. Drnec, R. Felici, and J. Frenken, J. Appl. Crystallogr. 48, 1324 (2015).

[30] C. Fourmental, A. Bellec, V. Repain, J. Lagoute, C. Chacon, Y. Girard, S. Rousset, Y. J. Dappe, A. Vlad, A. Resta, Y. Garreau, and A. Coati, Phys. Rev. Mater. 3, 083603 (2019).

[31] X. Lu, M. Grobis, K. H. Khoo, S. G. Louie, and M. F. Crommie, Phys. Rev. Lett. 90, 096802 (2003).

[32] J. P. Perdew, K. Burke, and M. Ernzerhof, Phys. Rev. Lett. 77, 3865 (1996).

[33] P. Giannozzi, S. Baroni, N. Bonini, M. Calandra, R. Car, C. Cavazzoni, D. Ceresoli, G. L. Chiarotti, M. Cococcioni, I. Dabo, A. Dal Corso, S. de Gironcoli, S. Fabris, G. Fratesi, R. Gebauer, U. Gerstmann, C. Gougoussis, A. Kokalj, M. Lazzeri, L. Martin-Samos, N. Marzari, F. Mauri, R. Mazzarello, S. Paolini, A. Pasquarello, L. Paulatto, C. Sbraccia, S. Scandolo, G. Sclauzero, A. P. Seitsonen, A. Smogunov, P. Umari, and R. M. Wentzcovitch, J. Phys.: Condens. Matter 21, 395502 (2009).

[34] P. Giannozzi, O. Andreussi, T. Brumme, O. Bunau, M. Buongiorno Nardelli, M. Calandra, R. Car, C. Cavazzoni, D.
Ceresoli, M. Cococcioni, N. Colonna, I. Carnimeo, A. Dal Corso, S. de Gironcoli, P. Delugas, R. A. DiStasio, A. Ferretti, A. Floris, G. Fratesi, G. Fugallo, R. Gebauer, U. Gerstmann, F. Giustino, T. Gorni, J. Jia, M. Kawamura, H.-Y. Ko, A. Kokalj, E. Küçükbenli, M. Lazzeri, M. Marsili, N. Marzari, F. Mauri, N. L. Nguyen, H.-V. Nguyen, A. Otero-de-la Roza, L. Paulatto, S. Poncé, D. Rocca, R. Sabatini, B. Santra, M. Schlipf, A. P. Seitsonen, A. Smogunov, I. Timrov, T. Thonhauser, P. Umari, N. Vast, X. Wu, and S. Baroni, J. Phys.: Condens. Matter 29, 465901 (2017).

[35] D. Vanderbilt, Phys. Rev. B 41, 7892 (1990).

[36] A. Dal Corso and A. Mosca Conte, Phys. Rev. B 71, 115106 (2005).

[37] D. Li, A. Smogunov, C. Barreteau, F. Ducastelle, and D. Spanjaard, Phys. Rev. B 88, 214413 (2013).

[38] D. Li, C. Barreteau, M. R. Castell, F. Silly, and A. Smogunov, Phys. Rev. B 90, 205409 (2014).

[39] P. Błoński, A. Lehnert, S. Dennler, S. Rusponi, M. Etzkorn, G. Moulas, P. Bencok, P. Gambardella, H. Brune, and J. Hafner, Phys. Rev. B 81, 104426 (2010).

[40] M. Blanco-Rey, J. I. Cerdá, and A. Arnau, New J. Phys. 21, 073054 (2019).

[41] D. Li, C. Barreteau, S. L. Kawahara, J. Lagoute, C. Chacon, Y. Girard, S. Rousset, V. Repain, and A. Smogunov, Phys. Rev. B 93, 085425 (2016).

[42] H. Yang, A. D. Vu, A. Hallal, N. Rougemaille, J. Coraux, G. Chen, A. K. Schmid, and M. Chshiev, Nano Lett. 16, 145 (2016).

[43] J. Tersoff and D. R. Hamann, Phys. Rev. Lett. 50, 1998 (1983).

[44] J. Tersoff and D. R. Hamann, Phys. Rev. B 31, 805 (1985). 